\title{
Lignin reinforcement of urea-crosslinked starch films for reduction of starch biodegradability to improve slow nitrogen release properties under natural aerobic soil condition
}

\begin{abstract}
The urea-crosslinked starch (UcS) film has a major drawback of very rapid biodegradability when applied as slow release fertilizer in soil. Lignin reinforcement of the UcS was used to prepare composite films, aimed to reduce the starch biodegradability and slow the release of nitrogen in aerobic soil condition. Study results revealed that mineralization of the composite films was delayed from 6.40 to $13.58 \%$ more than UcS film. Inhibition of composite films mixing with soil, the Michaelis-Menten reaction rates for Ŭ-amylase were inhibited 1.72ï 2.03 times whereas the Michaelis-Menten reaction rates for manganese peroxidase were increased 1.07ï 1.41 times compared to UcS film. Saccharidesï glucose, maltose and maltotriose demonstrated that their rates of formation (zero-order reaction) and depletion (first-order reaction); both were slowed more in aerobic soil which received the composite films. Increasing of lignin in composite films, the acid to aldehyde ratios of vanillyl and syringyl phenols of the lignin declined from 1.18 to 1.17 ( 0.76\%) and $1.59 \ddot{1} 1.56(\sim 1.78 \%)$, respectively. The diffusivity of nitrogen was effectively slowed $0.66 \mathrm{i} 0.94$ times by the lignin in composite films and showed a ñFickian diffusionò mechanism (release exponent $\mathrm{n}=0.095 \mathrm{i}$ $0.143)$.
\end{abstract}

Keyword: Biodegradability; Lignin composite films; Michaelis-Menten kinetics; Ureacrosslinked starch 